Laser microprocessing of steel with radially and azimuthally polarized femtosecond vortex pulses

This article has been downloaded from IOPscience. Please scroll down to see the full text article.

2012 J. Opt. 14085601

(http://iopscience.iop.org/2040-8986/14/8/085601)

View the table of contents for this issue, or go to the journal homepage for more

Download details:

IP Address: 138.253.100.121

The article was downloaded on 09/07/2013 at 15:28

Please note that terms and conditions apply. 


\title{
Laser microprocessing of steel with radially and azimuthally polarized femtosecond vortex pulses
}

\author{
O J Allegre, W Perrie, S P Edwardson, G Dearden and K G Watkins \\ Laser Group, School of Engineering, University of Liverpool, Liverpool L69 3GQ, UK \\ E-mail: O.Allegre@liv.ac.uk
}

Received 3 April 2012, accepted for publication 26 June 2012

Published 24 July 2012

Online at stacks.iop.org/JOpt/14/085601

\begin{abstract}
The use of a liquid-crystal spatial light modulator (SLM) device to convert a linearly polarized femtosecond laser beam into a radially or azimuthally polarized vortex beam is demonstrated. In order to verify the state of polarization at the focal plane, laser induced periodic surface structures (LIPSS) are produced on stainless steel, imprinting the complex vectorial polarization structures and confirming the efficacy of the SLM in producing the desired polarization modes. Stainless steel plates of various thicknesses are micromachined with the radially and azimuthally polarized vortex beams and the resulting cut-outs are analysed. The process efficiency and quality of each mode are compared with those of circular polarization. Radial polarization is confirmed to be the most efficient mode for machining high-aspect-ratio (depth/width >3) channels thanks to its relatively higher absorptivity. Following our microprocessing tests, liquid-crystal SLMs emerged as a flexible off-the-shelf tool for producing radially and azimuthally polarized beams in existing ultrashort-pulse laser microprocessing systems.
\end{abstract}

Keywords: radial, azimuthal, polarization, ultrashort-pulse, laser microprocessing

(Some figures may appear in colour only in the online journal)

\section{Introduction}

The past two decades have seen significant developments in ultrashort-pulse laser microprocessing. Thanks to the ultrashort timescale on which laser energy is coupled to the material, sub-micron precision processing can be achieved with very little thermal damage [1-3]. As a result, industrial processes based on femtosecond and picosecond laser pulse durations are becoming increasingly widespread. Manufacturing applications include the very precise drilling of holes for fuel-injection nozzles [3], the processing of silicon wafers [4] and the precise machining of medical stent devices $[5,6]$.

Laser-material interactions are known to be strongly influenced by the fluence, pulse duration, wavelength and polarization of the incident laser beam [7-9]. In particular, polarization affects both the efficiency and the quality of ultrashort-pulse microprocesses, including helical drilling and cutting [9-12]. For example, drilling high-aspectratio (depth/diameter) microscopic holes in metal with linearly polarized ultrashort-pulse lasers produces anisotropic profiles [10, 11]. Circularly polarized beams are chosen for most laser manufacturing applications thanks to their isotropic properties [10], although they do not offer the best processing efficiencies [13]. The potential benefits of using radial and azimuthal polarizations for various laser processing applications have been studied theoretically for several years [13-15], leading to predictions that the use of a radially polarized beam could enhance the efficiency of the cutting process by more than $50 \%$ compared to circular polarization [13]. However, producing radially or azimuthally polarized beams has been difficult until recently, making it complicated to verify these claims experimentally [14, 15]. In the last few years, a range of methods has been developed to produce these modes of polarization. Recent experimental work using these methods confirmed the benefits 


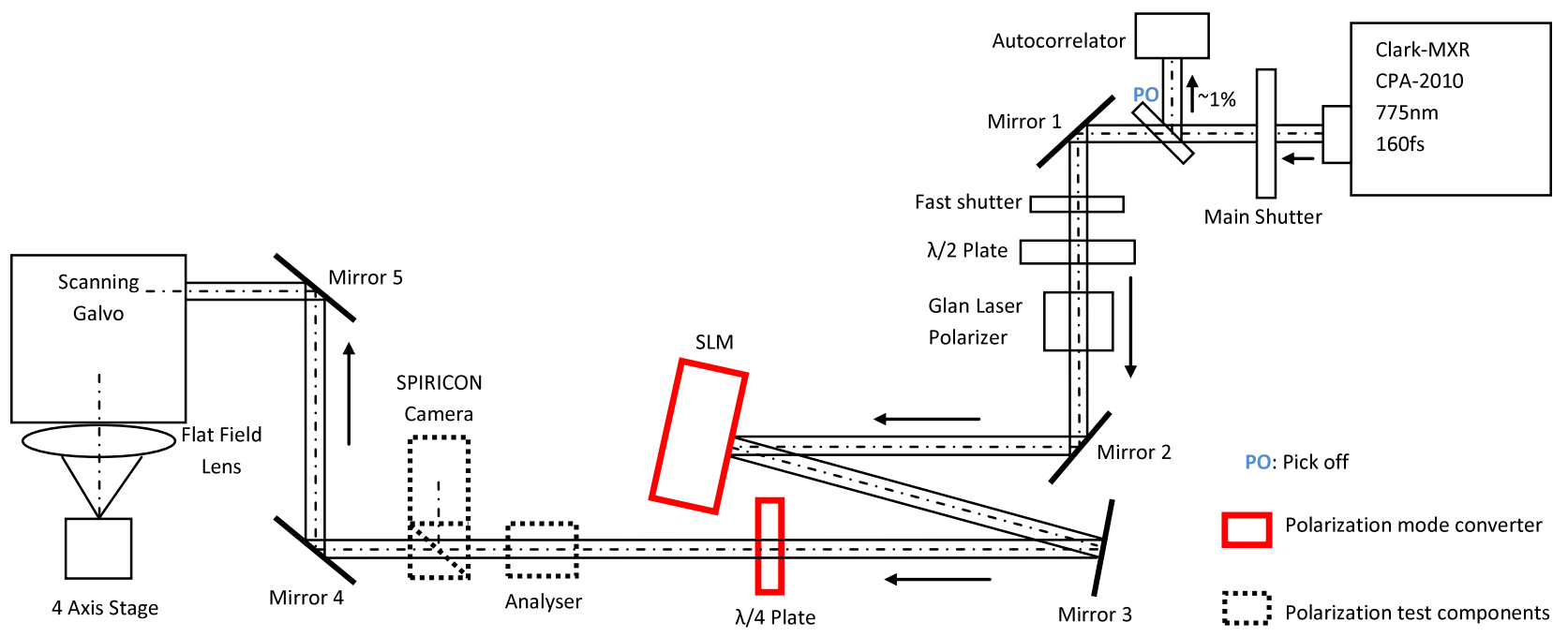

Figure 1. Experimental microprocessing setup showing how the 'polarization mode converter' is used. The 'polarization mode converter' consists of a SLM and a quarter-wave plate. Together these components convert the linearly polarized femtosecond laser beam into a radially, azimuthally or circularly polarized beam. The 'polarization test components' consist of a polarizing filter (analyser) and a SPIRICON (CCD) camera, used to verify the state of polarization of the beam. They are removed when the microprocessing tests are carried out.

of processing with radial or azimuthal polarization [16-20]. Unfortunately, these methods either required a cumbersome re-design of the laser cavity or used static extra-cavity polarization converters which sometimes lacked flexibility. Thanks to the latest technological developments in dynamic programmable liquid-crystal devices such as spatial light modulators (SLMs), it is now possible to produce such polarization modes in a more flexible and cost-effective manner. This approach has been used for high resolution microscopy applications in [21-23]. Modern SLMs are now able to sustain the high average power required for ultrashortpulse laser machining applications. Here, we demonstrate that these SLMs are well suited for producing radially or azimuthally polarized beams in a micromachining bench.

In this work, a phase-only liquid-crystal SLM is used to convert a linearly polarized femtosecond-pulse laser beam to radially or azimuthally polarized vortex beams in order to study the properties of these beams for microprocessing. A novel approach is used to visualize the complex vectorial polarization structures at the focal plane, using laser induced periodic surface structures (LIPSS) produced on stainless steel. This allows one to check if the expected state of polarization is achieved near the focal point. The micromachining properties of radially and azimuthally polarized beams are compared with those of circularly polarized beams. Micro-cutting tests are carried out on stainless steel, generating cut-outs of various depths and geometries. The resulting cutting profiles are characterized, demonstrating the effects that polarization has on the process.

\section{Experimental details}

\subsection{Experimental setup}

A schematic of the experimental setup is shown in figure 1. The output from a femtosecond laser (Clark-MXR CPA2010, with a minimum pulse width of $160 \mathrm{fs}, 775 \mathrm{~nm}$ central wavelength, spectral bandwidth of $5 \mathrm{~nm}, 1 \mathrm{~mJ}$ maximum pulse energy, $1 \mathrm{kHz}$ repetition rate and horizontal linear polarization) was attenuated by a half-wave plate and a Glan laser polarizer. The resulting linearly polarized beam was incident on the 'polarization mode converter', which consists of a Hamamatsu X10468-02 LCOS-SLM (liquid crystal on silicon spatial light modulator) and a zero-order quarter-wave plate. The SLM is of the phase-only, reflection type and enables spatially dependent control of the beam phase front. It consists of a $16 \mathrm{~mm} \times 12 \mathrm{~mm}, 800$ pixel $\times 600$ pixel array of horizontally oriented liquid-crystal phase retarders. In this paper, a vortex phase shift pattern was instigated by the SLM, producing the desired phase front. The quarter-wave plate was inserted after the SLM to convert this phase front into either a radial or an azimuthal polarization mode. The SLM and quarter-wave plate together act as a "polarization mode converter' and are referred to as such henceforth. As shown in figure 1, after reflection on Mirrors 4 and 5 the beam entered the $10 \mathrm{~mm}$ aperture of a scanning galvanometer system (made by GSI Lumonics) with a flat field lens $(f=100 \mathrm{~mm}$, $N A=0.03$ ), driven by a programmable PC interface board (Scanlab). Samples were mounted on a precision four-axis $(x, y, z, \theta)$ motion control system (Npaq system, produced by Aerotech) allowing accurate positioning of the sample in the focal plane.

\subsection{Polarization mode converter}

The Glan laser polarizer (see figure 1) produced a linearly polarized beam with its polarization oriented at $+45^{\circ}$ with respect to the (horizontal) liquid-crystal axis of the SLM. This linearly polarized beam can be described as a polarization vector with equal amplitude horizontal and vertical components. 

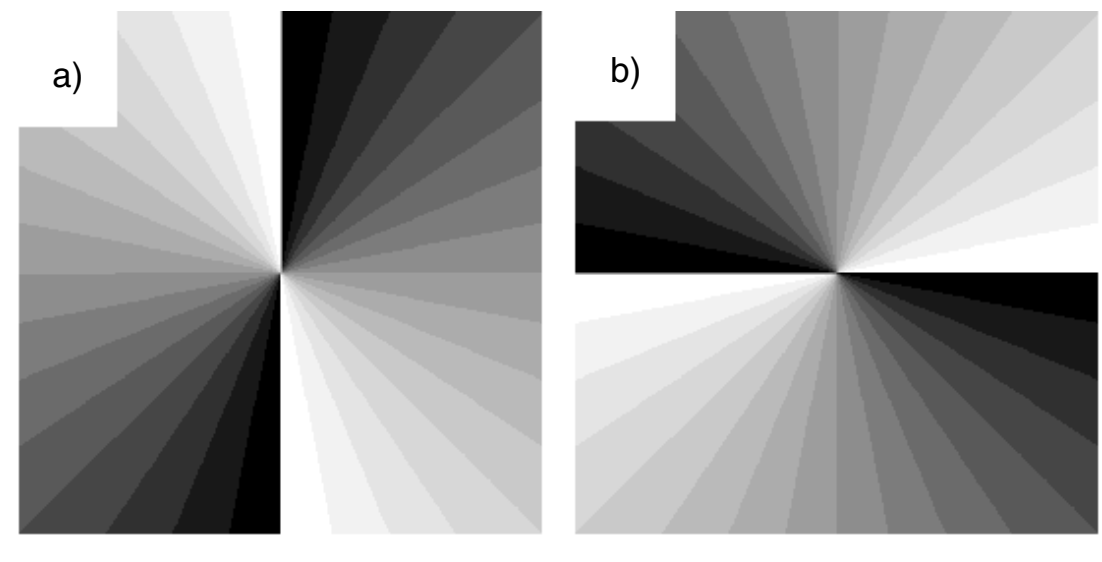

Figure 2. Vortex phase shift patterns generated by the SLM to convert a linear polarization into a radial polarization (pattern (a)) or an azimuthal polarization (pattern (b)).

Each pixel on the SLM generates a phase shift $\Phi_{x y}$ at the corresponding location $(x, y)$ on the beam cross-section. Therefore after reflection on the SLM, the horizontal component of the polarization vector was retarded by an amount $\Phi_{x y}$ relative to the vertical component. When $\Phi_{x y}=0$, the resulting polarization is linear (oriented at $+45^{\circ}$ ). When $\Phi_{x y}=\lambda / 4$, the resulting polarization is circular. For all other cases, the resulting polarization is elliptical. The beam was then transmitted through a quarter-wave plate with a fast axis oriented at $-45^{\circ}$ relative to the horizontal. This converted the elliptical polarization back to linear, with a rotation of $\Phi_{x y} / 2$ relative to the incident polarization. The Jones matrix representing the polarization properties of this system can be split into three terms:

$$
T_{x y}=Q \times\left[\begin{array}{cc}
1 & -1 \\
1 & 1
\end{array}\right] \times P_{x y} .
$$

The third term, $P_{x y}=\left[\begin{array}{ll}1 & 0 \\ 0 & \mathrm{e}^{\mathrm{i} \phi_{x y}}\end{array}\right]$, is the Jones matrix of one pixel at location $(x, y)$ on the pixel array of the SLM. The first term, $Q=\left[\begin{array}{ll}1 & 0 \\ 0 & \mathrm{e}^{-\mathrm{i} \frac{\pi}{2}}\end{array}\right]$, is the Jones matrix of the quarter-wave plate (see figure 1 ). When a linearly polarized beam with its polarization oriented at $+45^{\circ}$, represented by the Jones vector $\left(\begin{array}{l}1 \\ 1\end{array}\right)$ is incident on this setup, the resulting vector is

$$
T_{x y} \times\left(\begin{array}{l}
1 \\
1
\end{array}\right)=2 \mathrm{e}^{\mathrm{i} \frac{\Phi_{x y}-\pi}{2}} \times\left(\begin{array}{l}
\sin \frac{\Phi_{x y}}{2} \\
\cos \frac{\Phi_{x y}}{2}
\end{array}\right) .
$$

Equation (2) shows that the resulting vector is rotated by an angle $\Phi_{x y} / 2$ relative to the incident polarization. As $\Phi_{x y}$ is independently controllable for each pixel, the polarization mode converter enables a spatially dependent rotation of the polarization.

In this setup, an overall vortex phase pattern was created on the pixel array of the SLM by instigating a gradual change in the phase shift between adjacent pixels. $\Phi_{x y}$ varies from 0 to $4 \pi$ depending on the $(x, y)$ coordinates, producing a $4 \pi$ pitch vortex phase overall. When the resulting beam passed through the quarter-wave plate, a radial or azimuthal polarization was generated as described in (2). Equation (2) also shows that a residual $2 \pi$ phase vortex is still present after the quarter-wave plate: $\exp \left(i \Phi_{x y} / 2\right)$. By adding or subtracting a constant phase term $\pi$ to the overall phase vortex, the output polarization mode can be changed between radial and azimuthal (figure 2).

The linear-to-radial/azimuthal mode conversion was checked using a polarization analyser and a SPIRICON camera placed in the beam path, after the polarization mode converter (dotted components in figure 1). The beam profiles transmitted through the polarization analyser can be seen in figure 3 . The regions of the beam with polarization vectors that are parallel to the direction of the polarization analyser are transmitted through the analyser and are detected by the camera. The regions where the polarization vectors are perpendicular to the analyser are blocked. The beam profiles shown in figure 3 are consistent with what is typically expected from analysing a radially (figure 3(a)) or azimuthally (figure 3(b)) polarized beam, in line with [24]. As anticipated with such beams, rotating the polarization analyser clockwise causes the detected beam profiles to rotate clockwise.

\section{Results and discussion}

\subsection{Surface processing}

The results described above confirm that collimated beams with the expected polarizations have been generated by the SLM mode converter. However, the complex polarization and phase structures associated with radial/azimuthal vortex beams are also expected to affect the properties of the focal spots produced with these beams, as suggested in a numerical model in [25]. Therefore, a second check was required to confirm that the integrity of the polarization modes is maintained at the focal point of the system. Analysing the polarization in the focal region of an optical setup is extremely difficult, especially in the case of a femtosecond laser system, where the high peak intensity could damage any optics placed near the focal plane. To overcome this problem we used an indirect method, which involved producing LIPSS on a stainless steel sample, to analyse the polarization vectors in the focal plane. LIPSS are produced by laser irradiation of 

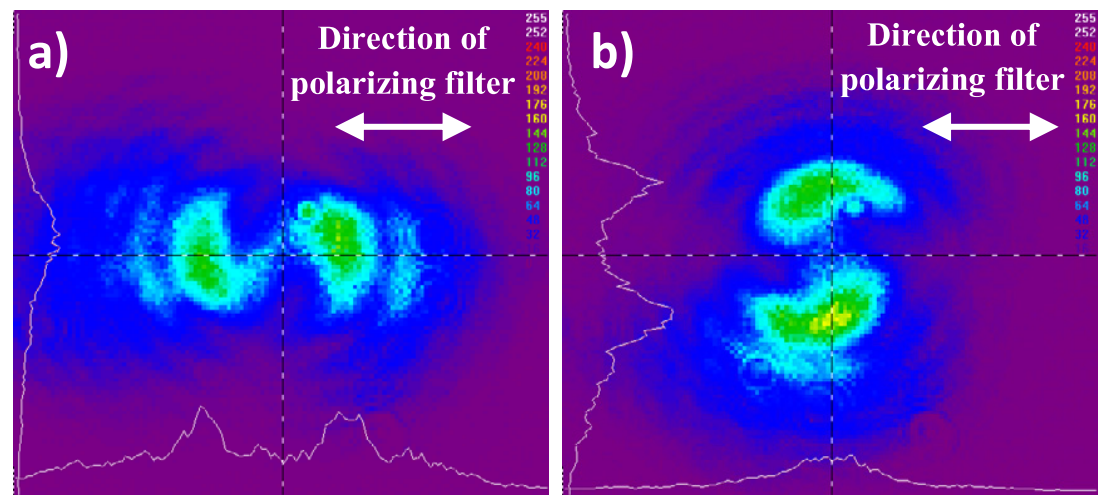

c)

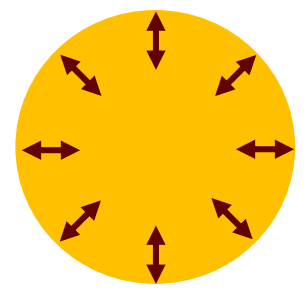

d)

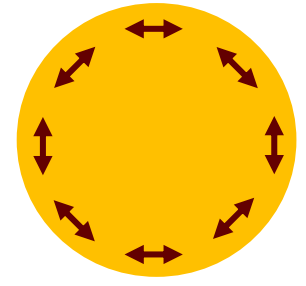

Figure 3. Beam profiles observed with a CCD camera (SPIRICON beam profiler) after transmission through a horizontally oriented polarization analyser. The colour-coded scale (in arbitrary units) represents the amplitude of the beam. Only the components of the polarization vectors that are parallel to the analyser are transmitted through. (a) The profile of a radially polarized beam; (b) the profile of an azimuthally polarized beam. (c) A schematic of the vectorial structure of a radially polarized beam; (d) a schematic of the structure of an azimuthally polarized beam.
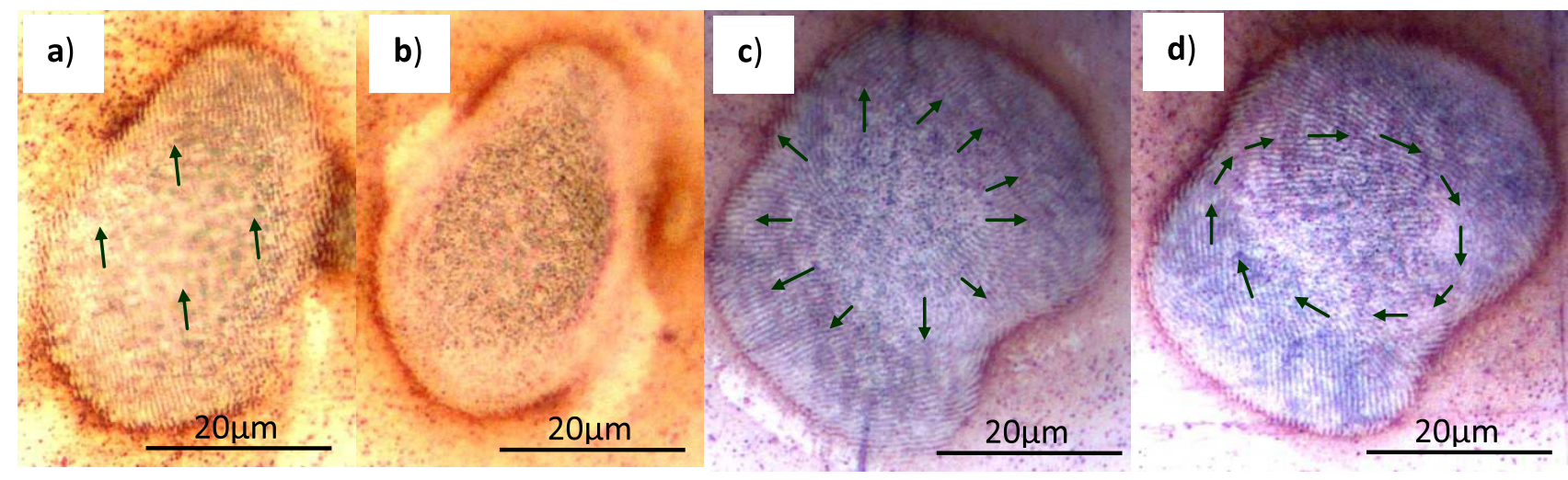

Figure 4. Optical micrographs showing the structures of the polarization vectors at the focal point of the microprocessing setup, imprinted into the surface of a stainless steel work-piece using LIPSS produced by $15 \pm 5$ fs pulses at $5 \mu \mathrm{J}$ per pulse $\left(1.5 \mathrm{~J} \mathrm{~cm}^{-2}\right)$. The arrows show the direction of the LIPSS and are perpendicular to that of the polarization vectors. The mode converter successively produced laser beams polarized linearly, circularly, radially and azimuthally, producing the laser spots in (a), (b), (c) and (d), respectively.

a material close to its ablation threshold. They develop in a direction perpendicular to that of the polarization vectors of the beam [26-29]. In this work, LIPSS are used to imprint the vectorial structure of the polarization near the focal point on the surface of the stainless steel sample.

The mode converter produced a linearly, circularly, radially and azimuthally polarized beam in turn. To produce the LIPSS, the surface of a stainless steel work-piece was marked with the laser pulse energy attenuated to $5 \mu \mathrm{J}$ $\left(1.5 \mathrm{~J} \mathrm{~cm}^{-2}\right.$ ), using the setup described in figure 1 (without the dashed components). The numerical aperture of the focusing optics is 0.03 . Each point was exposed to $\sim 15$ laser pulses, producing spot diameters of $38 \pm 10 \mu \mathrm{m}$. After processing, we analysed the laser marked spots with an optical microscope.
Figure 4(a) shows the LIPSS within the laser spot produced with a linearly polarized beam. The LIPSS formed as recurring linear structures oriented perpendicular to the direction of polarization. This is consistent with the experimental results described in [26]. Figure 4(b) shows the laser spot produced with a circularly polarized beam. No structure could be clearly identified within the spot. This is consistent with the fact that the direction of the polarization vectors varies over time in a circularly polarized beam. Figure 4(c) shows the laser spot produced with a radially polarized beam. The resulting LIPSS formed recurring curved structures centred on the middle of the laser spot. As the direction of the LIPSS is perpendicular to that of the electric field vectors [27], the geometry of the structures inside the laser spot implies that the electric field vectors are 
azimuthally oriented, i.e. the polarization is azimuthal around the focal point. Figure 4(d) shows the laser spot produced with an azimuthally polarized beam. The LIPSS formed an approximate circular pattern around the centre of the laser spot, implying a radial polarization near the focal point. As expected, the radially and azimuthally polarized beams produce patterns of LIPSS that are perpendicular to each other. This is most visible at the edges of the laser spots (figures 4(c) and (d)).

The investigation of the LIPSS above revealed that the radially polarized collimated beam from the mode converter produced an azimuthally polarized focal region and vice versa. It is interesting to consider why the state of polarization in the focal region is orthogonal to that of the collimated beam. The numerical simulation of a microscopy setup in [30] predicted such inversion of polarization, where a radially polarized vortex beam becomes azimuthally polarized in the focal region of the microscope. To explain the physical reason behind this phenomenon, we developed an analytical model of the optical setup used in our experiments. A detailed description of the model will be published elsewhere. The model showed that the polarization inversion at the focal plane is related to the vortex phase of the beam and does not occur when focusing a beam with a planar phase (i.e. without vortex phase). For example if we integrate the vector field produced by focusing a radially polarized vortex beam, we can show that the radially polarized components of the vector field cancel each other out at the focal plane, whereas the azimuthally polarized components interfere constructively. Our model also showed that this type of beam does not produce $100 \%$ polarization purity at the focal plane, but rather a hybrid mode that includes a small circularly polarized region in the centre of the focal spot, and azimuthally polarized regions around the centre. It is also possible to remove the polarization inversion effect by adding a two-lens telescope after the mode converter to re-image the surface of the SLM to the back aperture of the galvanometer. Further experimental work using an optical setup designed in this way has demonstrated that the inversion of polarization at the focal plane can be avoided. The details of these experiments will be published elsewhere.

It is noted that the size and shape of the laser spots were also affected by the vortex phase structure of the radially and azimuthally polarized beams. The radially/azimuthally polarized vortex beams focused as elliptical spots with two lobes. This is consistent with theoretical predictions in [31], where the focusing properties of a radially polarized vortex beam are investigated using a numerical simulation. The linearly and circularly polarized beams, which do not have a vortex phase structure, produced slightly smaller spots without lobes $(32 \pm 5 \mu \mathrm{m}$ for linearly/circularly polarized beams versus $40 \pm 5 \mu \mathrm{m}$ for radially/azimuthally polarized vortex beams).

The analysis above confirmed that the polarization mode converter described in this paper enabled a radial or azimuthal polarization state to be produced at the focal plane, although this state of polarization was orthogonal to that of the collimated beam. These results are the first experimental evidence showing the inversion of polarization when focusing a radially or azimuthally polarized vortex beam. This is an important result for material processing, where only the state of polarization within the focal region is relevant. It highlights the need to check the state of polarization at the focal plane of a laser processing bench. In the machining tests described henceforth, we refer to the state of polarization at the focal plane only, i.e. not the polarization of the collimated beam.

\subsection{Micromachining}

Having established the ability to generate the desired modes of polarization as discussed above, we looked into the machining process efficiency and the quality achievable with them. The aim is to either verify or invalidate theoretical predictions that radial polarization significantly improves the machining efficiency compared with circular polarization [13]. Thus stainless steel plates were machined using the scanning setup described earlier (figure 1) and the resulting cut-outs were studied.

Each stainless steel test piece was machined with three square holes, one for each polarization mode (the SLM mode converter was set to produce either a radially, an azimuthally or a circularly polarized focal region). Apart from the polarization state, the same process parameters were used for each square so that the results can be compared. A range of plate thicknesses were used (200-380 $\mu \mathrm{m})$ to give an insight into the way these polarization modes influence the coupling of the laser energy to the stainless steel at various depths. The machining tests were repeated using various exposure durations to compare the efficiency of the cutting.

A laser pulse energy of $75 \mu \mathrm{J}$ was used, corresponding to a peak fluence of $\sim 24 \mathrm{~J} \mathrm{~cm}^{-2}$. This is much higher than the ablation threshold of stainless steel which is around $0.16 \mathrm{~J} \mathrm{~cm}^{-2}$ [32]. The laser spot diameter, calculated according to the parameters of the optical setup, is $2 \omega o=$ $4 \lambda M^{2} f /(\pi D)=28 \pm 1 \mu \mathrm{m}\left(M^{2}=1.3, f=100 \mathrm{~mm}, D=\right.$ $6 \mathrm{~mm})$. For all the tests, $650 \mu \mathrm{m}$ sided square beam paths were programmed into the scanning galvanometer and scanned as a continuous loop for the chosen exposure duration, generating square cut-outs in the centre of the beam path. After exposure to the laser beam, the stainless steel plate was placed in an ultrasonic bath, so that the ultrasonic waves would clean and remove the central cut-outs if the cutting had been successful. This method is used to compare the cutting efficiency, the average volume of ablation and the quality of machining between each polarization state.

3.2.1. Overall cutting efficiency. A comparative study of the cutting efficiency achievable with each polarization mode was undertaken by checking which of the three holes were successfully cut out from the plate for a given exposure duration. To find out the most efficient polarization mode, we look at which hole is produced first, i.e. the one with the shortest exposure time.

A $380 \mu \mathrm{m}$ thick plate was exposed to the beam for $9.5 \mathrm{~min}$ per hole. Subsequent exposure to ultrasonic waves successfully removed the cut-outs for all polarization modes. 
Table 1. Minimum laser exposure required for cutting through stainless steel plates of various thicknesses. Results based on cutting $650 \mu \mathrm{m}$ square holes using radial, azimuthal and circular polarizations (775 nm, $160 \mathrm{fs}$ pulses, $1 \mathrm{kHz}$ repetition rate, pulse energy: $75 \mu \mathrm{J}$ ).

\begin{tabular}{llll}
\hline $\begin{array}{l}\text { Plate thickness } \\
(\mu \mathrm{m})\end{array}$ & $\begin{array}{l}\text { Azimuthally polarized } \\
\text { focal region }(\mathrm{min})\end{array}$ & $\begin{array}{l}\text { Radially polarized focal } \\
\text { region }(\mathrm{min})\end{array}$ & $\begin{array}{l}\text { Circularly polarized } \\
\text { focal region }(\mathrm{min})\end{array}$ \\
\hline 380 & 9.5 & 8.5 & 9 \\
310 & 9 & 8 & 8.5 \\
200 & 4 & 4 & 4 \\
\hline
\end{tabular}

The experiment was repeated, shortening the laser exposure to 8.5 min per hole. In this case, only cut-outs machined with a radially polarized focal region were removed by the ultrasonic waves. Reducing exposure further led to the failure of cutting for all polarization modes. These experiments were repeated with a $310 \mu \mathrm{m}$ thick plate and produced the same results: a radially polarized focal region successfully produced cut-outs in the shortest exposure duration ( $8 \mathrm{~min}$ in this case). Next, the experiment was repeated with a $200 \mu \mathrm{m}$ thick plate exposed to the laser beam for $4 \mathrm{~min}$ per hole. In this case, all the polarization modes produced clear cut-outs. The same plate was processed again, but with the laser exposure reduced to 3.5 min per hole. In that case, the cutting failed for all the polarization modes. In the case of machining a $200 \mu \mathrm{m}$ thick stainless steel plate, this comparative study could not single out one of the polarization modes as more efficient than the others. Table 1 summarizes these experimental results for all the processing parameters tested.

These machining tests revealed that a radially polarized focal region is more efficient than a circularly or azimuthally polarized one for cutting deep structures such as the ones produced in the 310 and $380 \mu \mathrm{m}$ thick stainless steel plates. Under these experimental conditions, a radially polarized focal region reduces the minimum laser exposure duration by $\sim 5 \%$ compared to a circularly polarized one. However, the comparative advantage of machining with this mode disappears when the depth of the machined structures is reduced, as demonstrated by cutting through $200 \mu \mathrm{m}$ thick plates.

The higher efficiency obtained with a radially polarized focal region for machining high-aspect-ratio channels was predicted theoretically in [13] and confirmed in the experimental analysis above. This increased efficiency is related to the internal reflections that occur during the machining of the micro-channels. When the laser beam is radially polarized in the focal region, it produces internal reflections which are mostly p-polarized. When the focal region is azimuthally polarized, the internal reflections are mostly s-polarized. With circular polarization, the internal reflections are an average of p- and s-polarizations. For a typical high-aspect-ratio channel geometry, the angle of incidence on the sidewalls is around $80^{\circ}$. The corresponding value for the reflectivity of steel is around $47 \%$ for p-polarization and $94 \%$ for s-polarization [10]. Therefore, the intensity of light reflected from the walls during the machining is approximately twice as much with an azimuthal (s-) polarization compared with a radial (p-) polarization. This results in a higher loss of energy through the exit of the channels machined with an azimuthal polarization. On the other hand, a radial polarization increases the coupling of the laser beam energy to the sidewalls during the machining, compared with a circular or azimuthal polarization. However, when lower aspect-ratio channels (depth/width < 3) are machined, fewer internal reflections occur on the sidewalls. The influence of polarization on coupling the laser energy to the work-piece is not so critical in that case, explaining the similarity in the efficiency of cutting through a $200 \mu \mathrm{m}$ thick plate between all the polarization modes.

It is noted that, due to the phase vortex of the radially/azimuthally polarized beams, the polarization purity at the focal plane is not $100 \%$. A residual circularly polarized region is present in the centre of the focal spots produced with these beams. This could slightly reduce the gains in processing efficiency compared with a homogeneous circularly polarized beam. It is thought that use of a radially polarized beam with a planar phase could further increase the efficiency of the process, since such a beam produces a better polarization purity at the focal plane compared with a vortex beam. Future work will look at microprocessing using an optical setup re-designed to produce radially/azimuthally polarized beams with a planar phase. It is also noted that the slight difference in the laser spot size between the radially/azimuthally polarized vortex beams and the circularly polarized one (figure 4) could also affect the process efficiency. This is discussed further in section 3.2.2.

3.2.2. Ablation efficiency. To further investigate the machining efficiency, the overall volume of ablated material was measured for each polarization mode. The cut-outs removed after machining as described above were imaged with an optical microscope. Table 2 (second row) shows optical micrographs of the sidewall of cut-outs removed from a $310 \mu \mathrm{m}$ thick stainless steel plate after a 9 min exposure to the laser beam per hole.

By measuring the cross-sectional area of the sidewalls of the cut-outs, the overall volume of ablated material can be derived for each polarization mode. Figure 5 shows the typical geometry of a cut-out and the dimensions required to derive the volume of ablated material. The cross-sectional area of the cut groove is $A_{\mathrm{G}}=H L-A_{\mathrm{CO}}$ (see figure 5). $H$ is the thickness of the sample plate $(H=310 \mu \mathrm{m}$ in the example illustrated in table 2). $L$ is the length of a side of the (square) beam path (650 $\mu \mathrm{m}$ here). $A_{\mathrm{CO}}$ is the cross-sectional area of a side of the cut-out. It is measured from the optical micrographs using the associated imaging software. The volume of ablated material $V$ can be derived by multiplying the cut groove cross-sectional area, $A_{\mathrm{G}}$, by the overall length of the groove, $V=4 L A_{\mathrm{G}}$. 
Table 2. Results of laser cutting square shaped holes from a $310 \mu \mathrm{m}$ thick stainless steel plate, using radial, azimuthal and circular polarizations $\left(775 \mathrm{~nm}, 160 \mathrm{fs}\right.$ pulses, $1 \mathrm{kHz}$ repetition rate, laser scanning speed: $5 \mathrm{~mm} \mathrm{~s}{ }^{-1}$, pulse energy: $75 \mu \mathrm{J}$, duration of laser exposure: 9 min per hole).

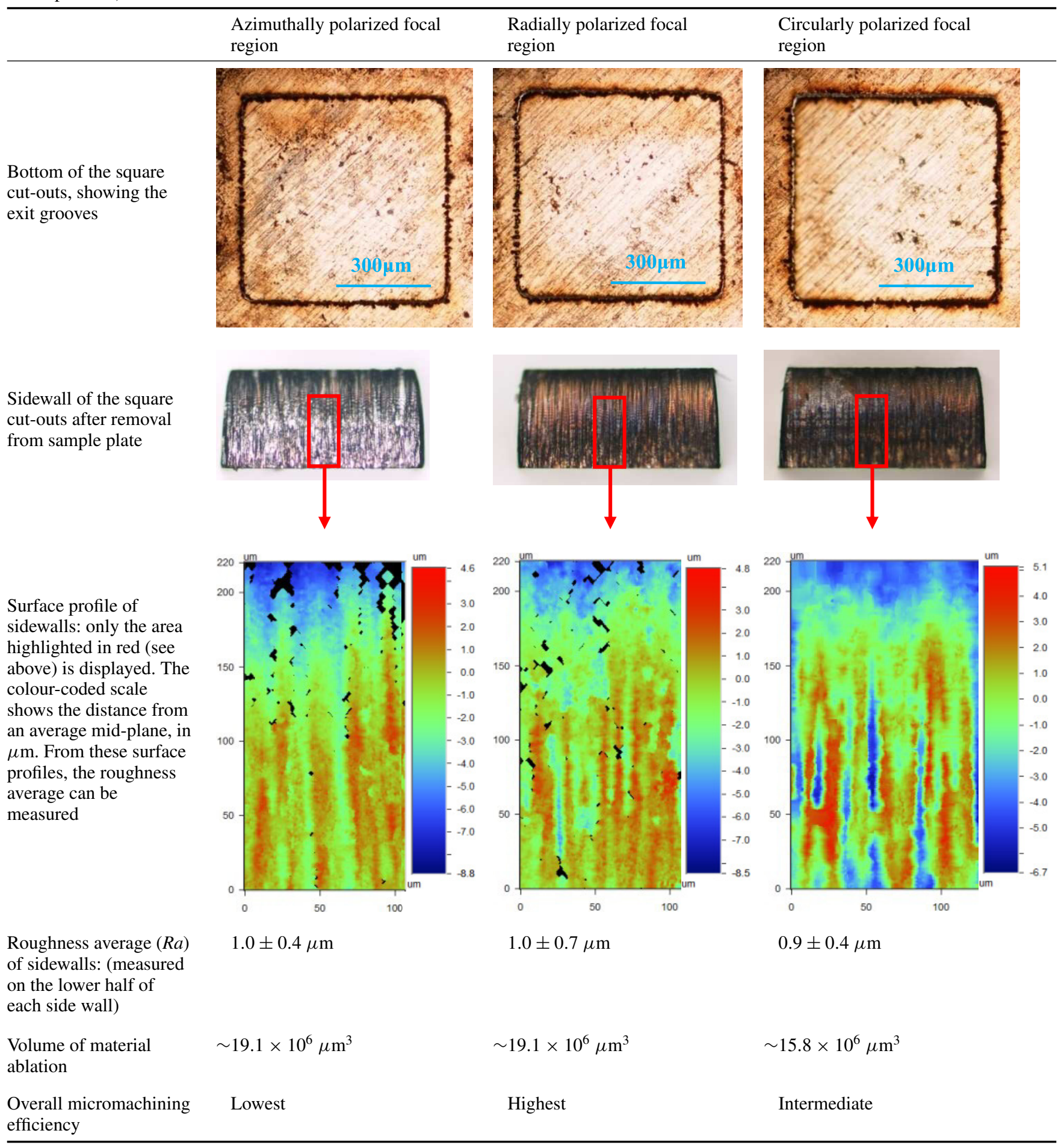

To ensure consistency in the results and gauge the level of uncertainty in the measurements, several cut-outs have been machined and measured for each polarization state. When $310 \mu \mathrm{m}$ thick stainless steel plates were exposed to the laser beam for 9 min per hole, a circularly polarized focal region ablated an average volume of $\sim 15.8 \times 10^{6} \mu^{3}$, while a radially or an azimuthally polarized focal region both ablated an average volume of $\sim 19.1 \times 10^{6} \mu \mathrm{m}^{3}$ (see table 2). According to these figures, radially and azimuthally polarized beams ablated approximately $20 \%$ more material than a circularly polarized one. The difference in ablation efficiency between these beams can be explained with the difference in the size of the laser spots at the focal point. 


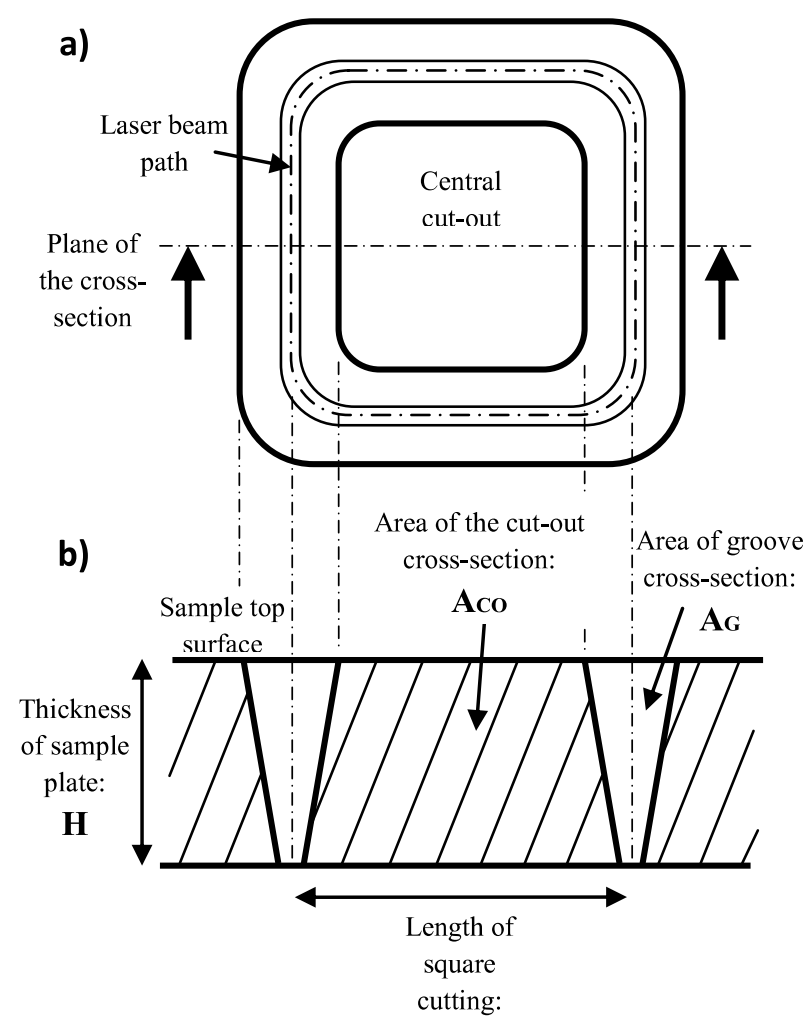

$\mathbf{L}$

Figure 5. Schematic of the geometry of a cut-out from a sample plate after laser exposure: (a) top view; (b) side cross-section.

When the mode converter was used to produce a radially or an azimuthally polarized vortex beam, the resulting laser spot at the focal point was wider than that produced with a circularly polarized planar phase beam $(32 \pm 5 \mu \mathrm{m}$ for linearly/circularly polarized beams versus $40 \pm 5 \mu \mathrm{m}$ for radially/azimuthally polarized vortex beams, see figure 4). A wider spot increases the size of the ablation front, enabling a more effective channelling of laser energy inside the machined structures by avoiding excessive reflections on the sidewalls. The wider spot also facilitates ejection of the ablated material, which helps to maintain a high ablation rate when machining high-aspect-ratio structures. The result of these effects is an increased sidewall taper angle. The circularly polarized beam, which produced a smaller spot at the focal point, ablated comparatively less material and produced a smaller taper.

3.2.3. Machining quality. In order to compare the quality of machining achieved with each polarization mode, the exit grooves obtained after laser cutting were examined with an optical microscope, prior to removing the cut-outs in an ultrasonic bath. Once the cut-outs had been removed, their sidewalls were studied using an optical surface profiling system (i.e. a white light interferometer, WYCO NT1100) to examine the surface quality and measure the roughness average $(R a)$.

Table 2 (first row) shows optical micrographs of the exit grooves obtained from machining a $310 \mu \mathrm{m}$ thick stainless steel plate for $9 \mathrm{~min}$ per hole. It can be seen that machining with a circular polarization mode produced ripples and filaments on the edges of the exit grooves. An azimuthally polarized focal region produced a better quality than a radially or circularly polarized one, with fewer ripples and filaments distorting the shape of the exit grooves. These findings are consistent across the full range of stainless steel plate thicknesses and exposure times tested here.

In order to check if the ripples and filaments seen on the exit grooves also affect the sidewalls, the roughness average, $R a$, of the surface of the sidewalls was measured for each cut-out from a $310 \mu \mathrm{m}$ thick stainless steel plate. To ensure consistency in the results, several cut-outs have been measured and the results averaged: $R a=1.0 \pm 0.7 \mu \mathrm{m}$ for a radially polarized focal region, $R a=1.0 \pm 0.4 \mu \mathrm{m}$ for an azimuthally polarized focal region and $R a=0.9 \pm 0.4 \mu \mathrm{m}$ for a circularly polarized one (see table 2 ). This indicates that in terms of surface roughness, the machining quality was fairly similar (within 10\%) for all polarization modes.

\section{Conclusions}

In this paper, we have demonstrated the use of a phase-only liquid-crystal SLM to convert a $775 \mathrm{~nm}$ linearly polarized femtosecond-pulse laser beam into radially and azimuthally polarized vortex beams. We have also demonstrated the use of these beams for microprocessing stainless steel. By producing LIPSS on the stainless steel, the vectorial structure of these polarization modes has been permanently imprinted into the material. This novel method allows analysis of the focusing properties of radially and azimuthally polarized vortex beams, which is typically a difficult thing to do. Interestingly, the results produced here indicate that geometrical effects can lead to the inverting of the polarization state of cylindrically polarized vortex phase beams at the focal region, i.e. a radially polarized vortex beam becomes azimuthally polarized at the focal region and vice versa. The inversion of the polarization state when focusing a radially or azimuthally polarized vortex beam is an important result for material processing, where only the state of polarization within the focal region is relevant.

The properties of the radial and azimuthal polarization modes for micromachining stainless steel plates of various thicknesses have been studied and compared with those of circular polarization. Radial polarization at the focal plane was confirmed to be the most efficient mode for machining high-aspect-ratio channels due to the relatively higher absorptivity of this mode, leading to a better coupling of the laser energy to the sidewall during the machining compared with other modes of polarization.

To our knowledge, this work is the first detailed investigation looking at the microprocessing properties of radially and azimuthally polarized femtosecond vortex pulses. Following our microprocessing tests, liquid-crystal SLMs emerged as a flexible off-the-shelf tool for producing radially and azimuthally polarized beams in existing ultrashort-pulse laser microprocessing systems. Future work will investigate femtosecond-pulse laser micromachining of semiconductor and dielectric materials, using an SLM to 
produce these polarization modes. The machining efficiency of radially/azimuthally polarized vortex beams will also be compared with that of radially/azimuthally polarized beams with a planar phase.

\section{Acknowledgments}

The authors gratefully acknowledge the support of the EPSRC and the help of Professor Miles Padgett, who provided the SLM control software used in this research as well as that of Dr Mary Erlund for her editorial contribution.

\section{References}

[1] Breitling D, Föhl C, Dausinger F, Kononenko T and Konov V 2004 Drilling of metals Femtosecond Technology for Technical and Medical Applications: Topics Appl. Phys. vol 96, ed F Dausinger et al (Berlin: Springer) pp 131-56

[2] Dausinger F 2005 Machining of metals with ultrashort laser pulses: from fundamental investigations to industrial applications Proc. SPIE 5777 840-5

[3] Breitling D, Ruf A and Dausinger F 2004 Fundamental aspects in machining of metals with short and ultrashort laser pulses Proc. SPIE 5339 49-63

[4] Sudani N, Venkatakrishnan K and Tan B 2009 Laser singulation of thin wafer: die strength and surface roughness analysis if $80 \mu \mathrm{m}$ silicon dice Opt. Laser Eng. $47850-4$

[5] Rizvi N H 2003 Femtosecond laser micro-machining: current status and applications RIKEN Rev. 50 107-12

[6] Ostendorf A, Bauer T, Korte F, Howorth J R, Momma C, Rizvi N H, Saviot F and Salin F 2002 Development of an industrial femtosecond laser micro-machining system Proc. SPIE 4633 128-35

[7] Banks P S, Feit M D, Rubenchik A M, Stuart B C and Perry M D 1999 Material effects in ultra-short pulse laser drilling of metals Appl. Phys. A 69 S377-80

[8] Hahne S, Johnston B F and Withford M J 2007 Pulse-to-pulse polarization-switching method for high-repetition-rate lasers Appl. Opt. 46 954-8

[9] Föhl C and Dausinger F 2003 High precision deep drilling with ultrashort pulses Proc. SPIE 5063 346-5

[10] Nolte S, Momma C, Kamlage G, Ostendorf A, Fallnich C, von Alvensleben F and Welling H 1999 Polarization effects in ultrashort-pulse laser drilling Appl. Phys. A $68563-7$

[11] Allegre O J, Perrie W, Bauchert K, Liu D, Edwardson S P, Dearden G and Watkins K G 2012 Real-time control of polarisation in ultra-short-pulse laser micro-machining Appl. Phys. A 107 445-54

[12] Tönshoff H K, Momma C, Ostendorf A, Nolte S and Kamlage G 2000 Microdrilling of metals with ultrashort laser pulses J. Laser Appl. 12 23-7

[13] Niziev V G and Nesterov A V 1999 Influence of beam polarization on laser cutting efficiency J. Phys. D: Appl. Phys. 32 1455-61

[14] Stalder M and Schadt M 1996 Linearly polarized light with axial symmetry generated by liquid-crystal polarization converters Opt. Lett. 21 1948-50
[15] Iglesias I and Vohnsen B 2007 Polarization structuring for focal volume shaping in high-resolution microscopy Opt. Commun. 271 40-7

[16] Weber R, Michalowski A, Abdou-Ahmed M, Onuseit V, Rominger V, Kraus M and Graf T 2011 Effects of radial and tangential polarization in laser material processing Phys. Procedia 12 21-30

[17] Meier M, Romano V and Feurer T 2007 Material processing with pulsed radially and azimuthally polarized laser radiation Appl. Phys. A 86 329-34

[18] Venkatakrishnan K and Tan B 2006 Interconnect microvia drilling with a radially polarized laser beam J. Micromech. Microeng. 16 2603-7

[19] Kraus M, Ahmed M A, Michalowski A, Voss A, Weber R and Graf T 2010 Microdrilling in steel using ultrashort pulsed laser beam with radial and azimuthal polarization $O p t$. Express 18 22305-13

[20] Hnatovsky C, Shvedov V, Krolikowski W and Rode A 2011 Revealing local field structure of focused ultrashort pulses Phys. Rev. Lett. 106123901

[21] Yew E Y S and Sheppard C J R 2007 Second harmonic generation polarization microscopy with tightly focused linearly and radially polarized beams $\mathrm{Opt}$. Commun. $275453-7$

[22] Beversluis M R, Novotny L and Stranick S J 2006 Programmable vector point-spread function engineering Opt. Express 14 2650-6

[23] Yoshiki K, Hashimoto M and Araki T 2005 Three-dimensional polarization control and its application to SHG imaging Japan. J. Appl. Phys. 44 655-6

[24] Beresna M, Gecevicius M, Kazansky P G and Gertus T 2011 Radially polarized optical vortex converter created by femtosecond laser nanostructuring of glass Appl. Phys. Lett. 98201101

[25] Dong X, Gao X, Chen J and Zhuang S 2011 Radially polarized hollow Gaussian beam with on-axis spiral optical vortex Optik—Int. J.: Light Electron Opt. doi:10.1016/j. ijleo.2011.11.001

[26] Mellor L, Edwardson S, Perrie W, Dearden G and Watkins K 2009 Surface plasmon polaritons for micro and nano-texturing of metal surfaces Proc. ICALEO vol 102, pp 1329-37

[27] Guosheng Z, Fauchet P M and Siegman A E 1982 Growth of spontaneous periodic surface structures on solids during laser illumination Phys. Rev. B 26 5366-81

[28] Ursu I, Mihăilescu I N, Prokhorov A M, Tokarev V N and Konov V I 1987 High-intensity laser irradiation of metallic surfaces covered by periodic structures $J$. Appl. Phys. $612445-57$

[29] Nanai L, Vajtai R, Hevesi I, Laiho R and Heikkila L 1992 Periodic nanostructures observed by STM on vanadium surface preilluminated with a cw Yag-laser Superlatt. Microstruct. 11 435-8

[30] Kang H, Jia B and Gu M 2010 Polarization characterization in the focal volume of high numerical aperture objectives $\mathrm{Opt}$. Express 18 10813-20

[31] Machavariani G, Lumer Y, Moshe I and Jackel S 2007 Effect of the spiral phase element on the radial-polarization $(0,1)$ LG beam Opt. Commun. 271 190-6

[32] Mannion P, Magee J, Coyne E and O'Connor G M 2003 Ablation thresholds in ultrafast laser micro-machining of common metals in air Proc. SPIE 4876 470-8 